\title{
Optofluidic sensor engineering towards plutonium concentration measurements
}

T. Allenet, F. Geoffray, D. Bucci, L. Guillerme, F. Canto, et al.

T. Allenet, F. Geoffray, D. Bucci, L. Guillerme, F. Canto, A. Bouchard, J.-E. Broquin, "Optofluidic sensor engineering towards plutonium concentration measurements," Proc. SPIE 10106, Integrated Optics: Devices, Materials, and Technologies XXI, 101060U (22 February 2017); doi: 10.1117/12.2252190

SPIE. Event: SPIE OPTO, 2017, San Francisco, California, United States 


\title{
Optofluidic sensor engineering towards plutonium concentration measurements
}

\author{
T. Allenet ${ }^{\mathrm{a}}$, F. Geoffray ${ }^{\mathrm{b}}$, D. Bucci ${ }^{\mathrm{a}}$, L. Guillerme ${ }^{\mathrm{b}}$, F. Canto ${ }^{\mathrm{b}}$, A. Bouchard ${ }^{\mathrm{a}}$, J-E. Broquin ${ }^{\mathrm{a}}$ \\ ${ }^{a}$ Univ. Grenoble Alpes, IMEP-LAHC, F-38000 Grenoble, CNRS, IMEP-LAHC, F-38000 Grenoble, \\ France \\ ${ }^{\mathrm{b}}$ CEA Nuclear Energy Division, Research Department on Mining and Fuel Recycling Processes, \\ Marcoule F-30207 Bagnols-sur-Cèze, France
}

\begin{abstract}
Research in nuclear safety and fuel reprocessing has led to a surging need for novel chemical analysis tools with reduced analyte and effluent volumes. Recent technological advances for the elaboration and packaging of glass optofluidic cointegrated sensors have opened up the way for said analys is in harsh environments. We discuss a sensor engineering approach for the construction of an integrated absorption spectrometer with an ion-exchange core.

$\mathrm{Pu}(\mathrm{VI})$ oxidation state exhibits a major absorption peak at a wavelength of $831 \mathrm{~nm}$ with a molar absorption coefficient of $545 \mathrm{~L} \mathrm{~mol}^{-1} \cdot \mathrm{cm}^{-1}$. An evanescent waveguiding sensing structure that allows guided fluid/light interaction is investigated in order to provide absorption spectroscopy measurements. The work presented consists of optical simulations as well as experimental measurements. Waveguide engineering with respects to modal transmission, field/fluid interaction coefficient $\Gamma$ and device losses is presented. The simulations are carried out by computing ion-exchanged waveguide refractive index distribution and using it in mode solver software. Device optical characterization and bench tests are carried out to verify approach viability. First device measurements of a neodymium absorption peak in nuclear manipulation conditions are displayed.
\end{abstract}

Keywords: Optical engineering, microfluidics, opto-fluidics, chemical analysis, absorption spectroscopy, harsh environment

\section{INTRODUCTION}

Nuclear power is an important means of energy production worldwide. With the growth of this industry comes the need for the development of nuclear recycling processes and nuclear safety. Current industrial fuel reprocessing techniques require chemical control along a fuel dismantlement chain. The harsh nature of such measurements ensues extremely heavy facility requirements in terms of operator and environmental safety. Nowadays, fuel separation chemistry takes place in hot cells with meter-thick leaded-glass windows operated by remote manipulators. An idea to face these fuel management is sues consists in reducing sample and effluents volumes, since radioactive dosage is proportional to sample volume $^{1}$. This should allow lightening infrastructure constraints while reducing breach risks, measurement delays and global costs.

The process of nuclearizing of a chemical analysis device faces the constraints listed below:

- Resistance to harsh environments (acidity, ionizing radiation, oxidizing environment)

- Reduced sample volume

- Use of a non-destructive and versatile means of probing

- Need for deported measurements by means of optical fibers

We present engineering of a sturdy borosilicate glass optical and fluidic integrated device that allows fluid and light interaction in order to provide absorption spectroscopy measurements. Device packaging was developed in order to allow deported measurements of the device inside a nuclear glove-box.

We have directed our study towards the detection of plutonium $\mathrm{VI}$ oxidized state $(\mathrm{Pu}(\mathrm{VI}))$ which exhibits an absorption peak at $831 \mathrm{~nm}$ shown in Figure 1. Absorption is particularly adapted for the sought-out application in addition to providing label-free detection of a range of elements. Neodymium(Nd) is used as a chemical model for its ease of use, being non radioactive. In this study, we focus on its absorption peak at $794 \mathrm{~nm}$, shown in Figure 1, for the design and characterization of the device. The goal of this work is therefore to provide a NIR-VIS absorption spectroscopy tool compatible with nuclear measurement constraints.

Integrated Optics: Devices, Materials, and Technologies XXI, edited by Sonia M. García-Blanco, Gualtiero Nunzi Conti, Proc. of SPIE Vol. 10106, 101060U · (C) 2017 SPIE

CCC code: $0277-786 \mathrm{X} / 17 / \$ 18 \cdot$ doi: $10.1117 / 12.2252190$ 

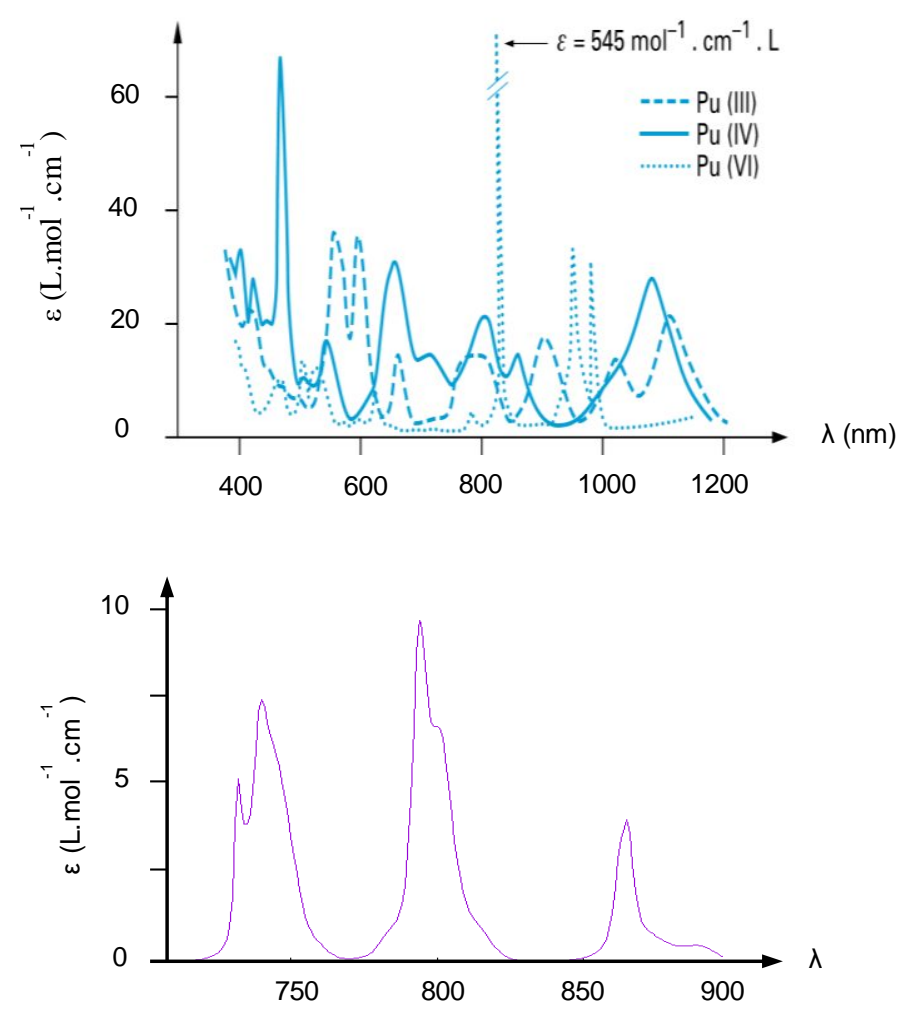

Figure 1. Absorption peak measurements of several plutonium oxidation states in $1 \mathrm{M}$ nitric acid ${ }^{2}$ (top) and of $\mathrm{Nd}$ (bottom).

In this paper, we will start by describing the device principle of operation before displaying waveguide engineering, fabrication and optical characterization of the co-integrated device. We will then discuss device packaging and chemical analysis results. Finally we will show first measurement results of the system in a nuclear environment.

\section{DEVICE PRINCIPLE OF OPERATION}

An evanescent-wave sensing design was chosen to seek out absorption spectroscopy ${ }^{3,4}$. Interaction between guided light and fluid is obtained in the evanescent part of a propagating mode in an adjacent fluidic channel according to Figure 2. This interaction is described by Beer-Lambert's law, describing light absorption $\mathrm{A}(\lambda)$ by a chemical element:

$$
A(\lambda)=-\log _{10} \frac{I_{L}}{I_{0}}=\Gamma \cdot C \cdot \varepsilon(\lambda) \cdot L
$$

with $\mathrm{I}_{0}$ (a.u.) the initial intensity, $\mathrm{I}_{\mathrm{L}}$ (a.u.) the trans mitted intensity after an interaction length of $\mathrm{L}$ (in cm), $\mathrm{C}$ (in mol. $\mathrm{L}^{-1}$ ) the chemical element's concentration, and $\varepsilon\left(\right.$ in $\left.\mathrm{L} \mathrm{mol}^{-1} . \mathrm{cm}^{-1}\right)$ the molar attenuation coefficient. $\varepsilon$ is specific to each chemical element and quantifies the affinity of a chemical to interact with light at a particular wavelength.

The device consists of two bonded wafers, the first containing the microfluidic function and the second containing an optical waveguide, as shown in Figure 3. The microfluidic function consists of a large central microfluidic channel which acts as the sensing window, and of 4 fluidic accesses designed to allow fluid insertion fromchip edges separate from optical input and output edges. The optical function consists of a waveguide core at the substrate's surface. The complete device therefore consists of two different waveguide cross-sections that we will refer to as "glass-encapsulated waveguide" and "evanescent waveguide". The fabrication of glass-integrated waveguides is achieved with the ionexchange technology which has been extensively reported ${ }^{5,6}$. Optical cores are elaborated by first depositing an aluminum-oxyde ion-opaque mas king layer which is patterned by photolithography to define waveguides apertures. The substrate is then plunged into a molten salt bath containing dopant ions. 
Evanescent field region

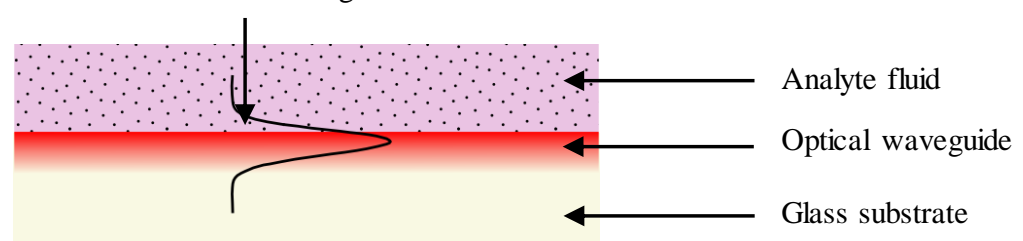

Figure 2. Evanescent-wave sensing principle. Light and fluid interaction is obtained in the evanescent part of the propagating mode in an adjacent fluidic channel. Analyte in Encapsulated waveguide

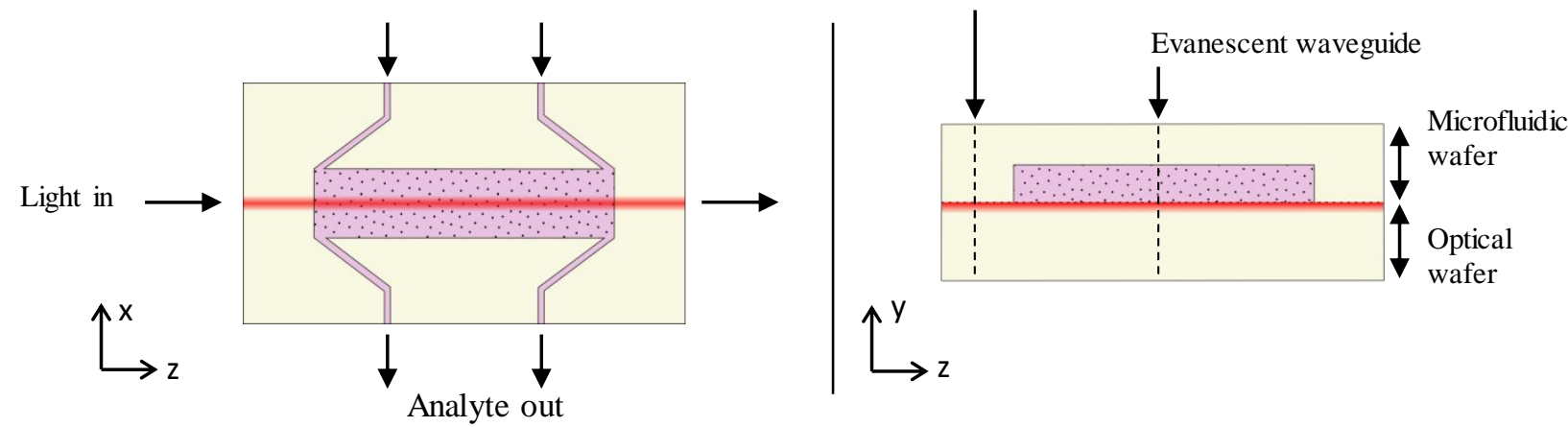

Figure 3. Cross-sectional views of the sensor. An optical and a microfluidic wafer are bonded in order to obtain a monolithic co-integrated evanescent wave sensing structure.

The substitution and diffusion of dopant ions into the glass matrix results in a local refractive index increase. After removal of the masking layer we therefore obtain an integrated gradient-index optical core at the substrate's surface, due to dopant ion difference in polarisability and ionic radius. Key parameters of the ion-exchange process are the choice of exchanged ions that impact refractive index gradient, exchange temperature that affects ion diffusion coefficients, waveguide aperture and exchange duration which determine both the depth and width of the optical core. Here, we will use previously investigated and commercially available Schott BF33 borosilicate glass containing $4 \%$ of sodium and potassium ions $(\mathrm{Na}+$ and $\mathrm{K}+)$ to proceed to a silver/sodium ion exchange $(\mathrm{Ag}+/ \mathrm{Na}+)$.

\section{WAVEGUIDE ENGINEERING}

Computer simulations were set up to model refractive index profiles of the ion-exchanged waveguides and their optical response. Waveguide design was chosen with respects to three key-factors:

- Single-mode behavior between 700 and $900 \mathrm{~nm}$

- Interaction coefficient

- Device coupling losses

In the following computational approach, we will describe the simulations with regards to the points listed above.

\subsection{Simulation protocol}

Ion-exchange modelization has been put forward in the literature ${ }^{5}$. In order to model ion-exchanged waveguide optical behavior, doping ion concentration distribution $\mathrm{C}(\mathrm{x}, \mathrm{y})$ was obtained by computing the diffusion equations with a finite difference solver based on ion diffusion coefficients, waveguide aperture, exchange temperature and duration. The obtained profiles were used to calculate the refractive index variation profile $\Delta \mathrm{n}(\mathrm{x}, \mathrm{y})$ ensued by the ion-exchange. A commercial scalar mode solver developed by OptiWave was used to simulate modal response of the encapsulated waveguides $\left(\mathrm{n}_{\text {sup }}=\mathrm{n}_{\text {sub }}\right)$ and the evanescent waveguides $\left(\mathrm{n}_{\text {sup }}=1.333\right)$. Figure 4 maps out the simulation protocol. Optical behavior of the $\mathrm{Ag}^{+} / \mathrm{Na}^{+}$ion-exchanged waveguides was studied as a function of aperture a and ion-exchange duration $\mathrm{t}$ at $353^{\circ} \mathrm{C}$. The influence of the bonding process' annealing at $150^{\circ} \mathrm{C}$ during $72 \mathrm{~h}$ was also taken into consideration. 


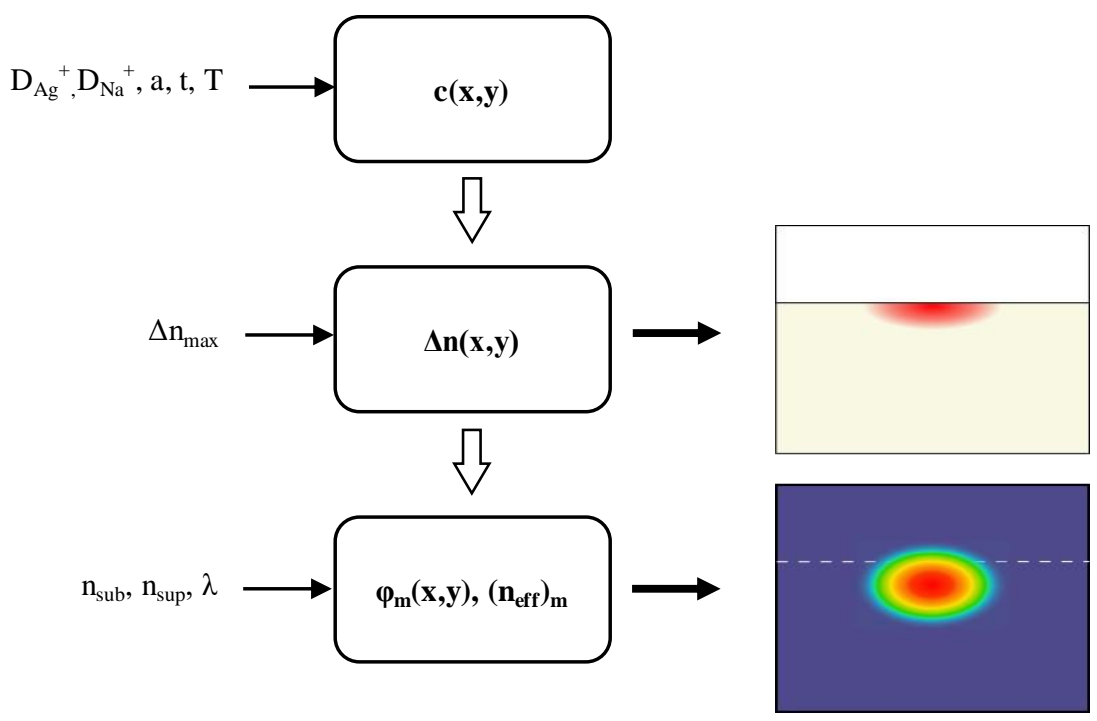

Figure 4. Simulation protocol with input parameters on the left and output file examples on the right.

\subsection{Broad-band single-mode propagation}

Broad-band light confinement propagation is sought out between 700 and $900 \mathrm{~nm}$. It is essential to achieve single-mode propagation in order to insure spectrum stability by avoiding modal interferences. Evanescent and encapsulated waveguide modal response was studied as a function of wavelength in order to assess mode cut-off wavelengths. Figure 5 shows the variation of mode refractive indices as a function of wavelength for both the encapsulated and evanescent waveguide. Simulations show an increase in effective indices of the modes with exchange duration and waveguide aperture. We also witness a decrease in confinement at high wavelengths and appearance of higher order modes at smaller wavelengths. Due to its higher symmetry, the encapsulated waveguide shows better light confinement than the evanescent waveguide that can therefore be used as a modal filter. Single modal broad-band propagation is achievable in both waveguiding structures as is shown in Figure 6.
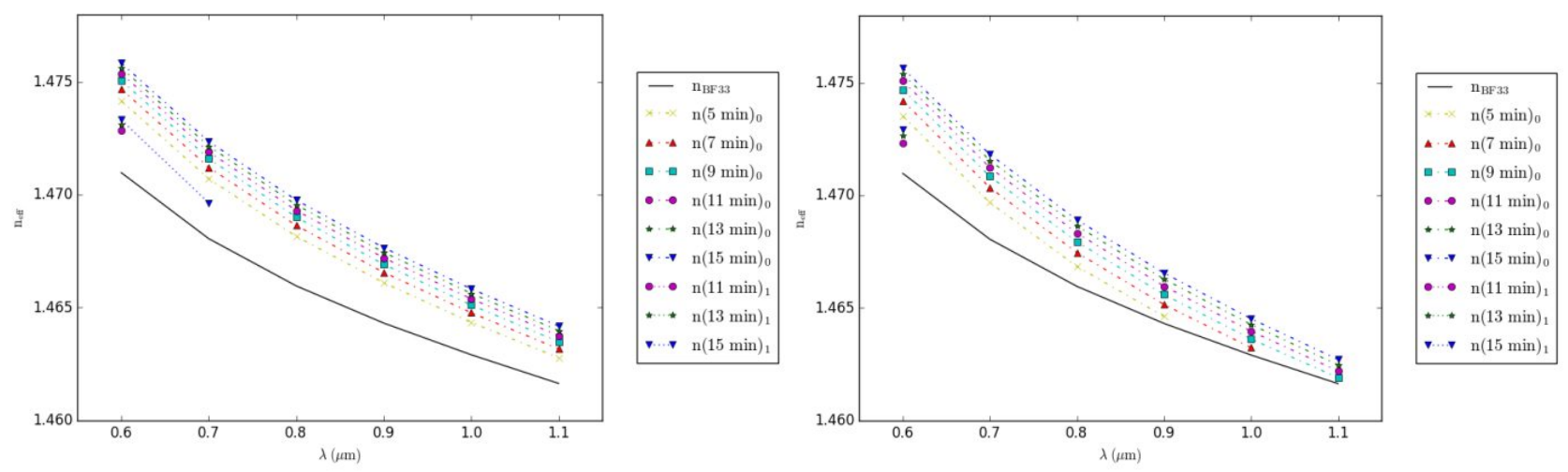

Figure 5. Variation of the effective index with respect to wavelength for the encapsulated (left) and evanescent (right) waveguides. Waveguide has an aperture of $3 \mathrm{um}$ and was annealed $72 \mathrm{~h}$ at $150^{\circ} \mathrm{C}$. Substrate refractive index $\left(\mathrm{n}_{\mathrm{BF} 33}\right)$ is shown in order to show design limits. 

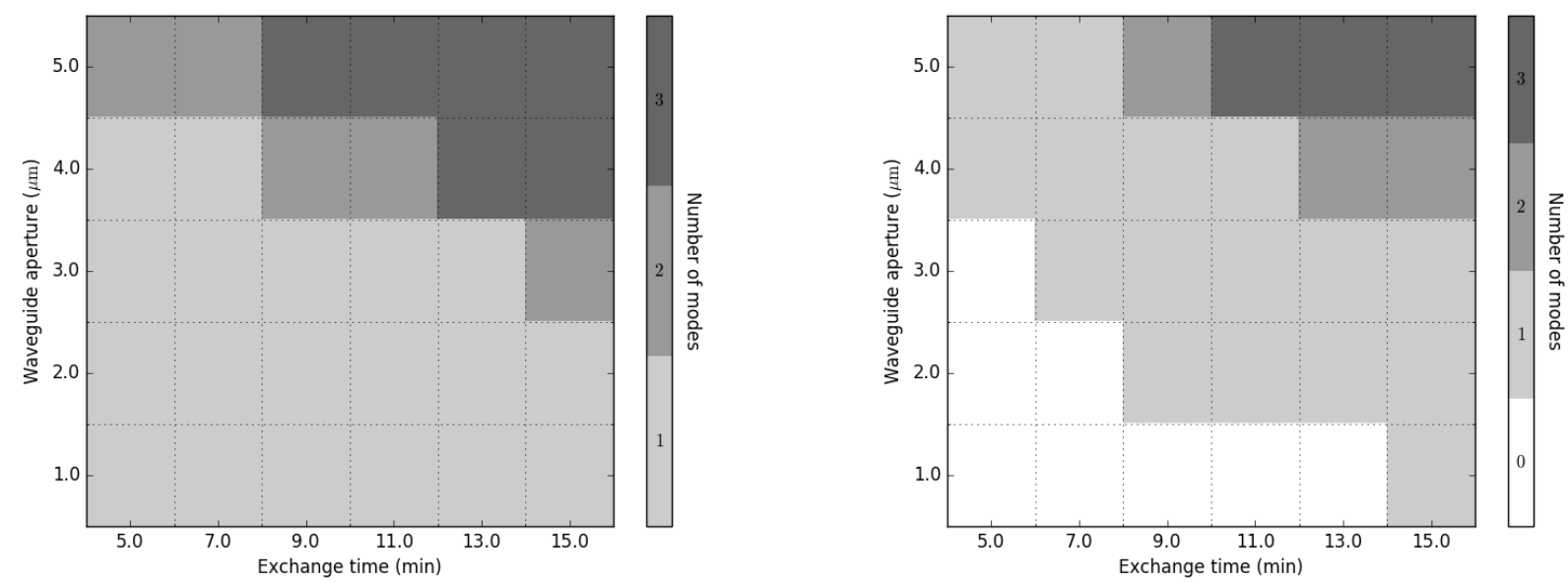

Figure 6. Number of modes of the encapsulated (left) and evanescent (right) waveguide over the 700-1000 nm wavelength range. " 0 " indicated that none of the modes can propagate over the entire range of interest.

\subsection{Interaction coefficient}

Waveguide interaction coefficient was studied in order to assess chemical measurement detection limits. We define the interaction coefficient of an optical mode of $\mathrm{m}^{\text {th }}$ order as:

$$
\Gamma_{\mathrm{m}}=\frac{\iint_{\Omega}\left|\varphi_{\mathrm{m}}\right|^{2} \mathrm{dxdy}}{\iint_{\mathbb{R}^{2}}\left|\varphi_{\mathrm{m}}\right|^{2} \mathrm{dxdy}}
$$

where $\Omega$ represents the analyte fluid. Given that we are interested in single-mode propagation here, we will only consider fundamental modes $(\mathrm{m}=0)$ and their interaction coefficients. The evolution of $\Gamma$ at $794 \mathrm{~nm}$ is shown in Figure .

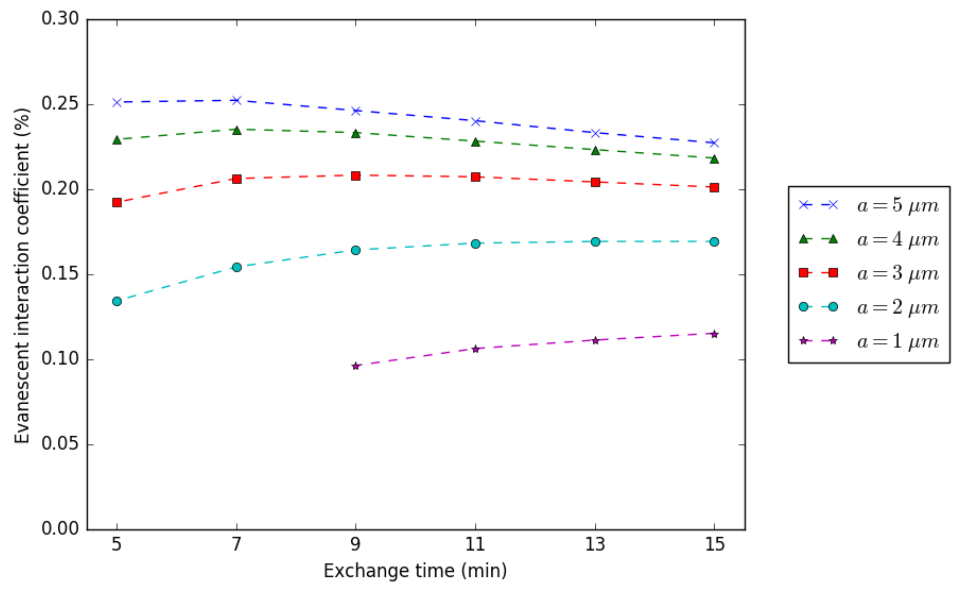

Figure 7. Simulation results of evanescent interaction coefficient at $\lambda=794 \mathrm{~nm}$.

Results show that the interaction coefficient increases with waveguide aperture and undergoes scarce relative variations for exchange durations higher than $7 \mathrm{~min}$. For example, for an aperture of $a=3 \mu \mathrm{m}$, we obtain that the interaction coefficient varies from $0.19 \%$ to $0.22 \%$ when the exchange time ranges from 5 to $15 \mathrm{~min}$. 


\subsection{Coupling losses}

It is of paramount importance to assess signal losses of an optical sensor in addition to its sensitivity. Intrinsic coupling losses between the different sections of the detector were assessed. Considering the integrated sensor with a microfluidic channel, coupling losses occur at the evanescent/encapsulated interfaces as well as at the chip edges between the optical fiber and encapsulated waveguide modes. Figure 8 illustrates field overlap integrals of each transition as well as total field overlap integral at $800 \mathrm{~nm}$.

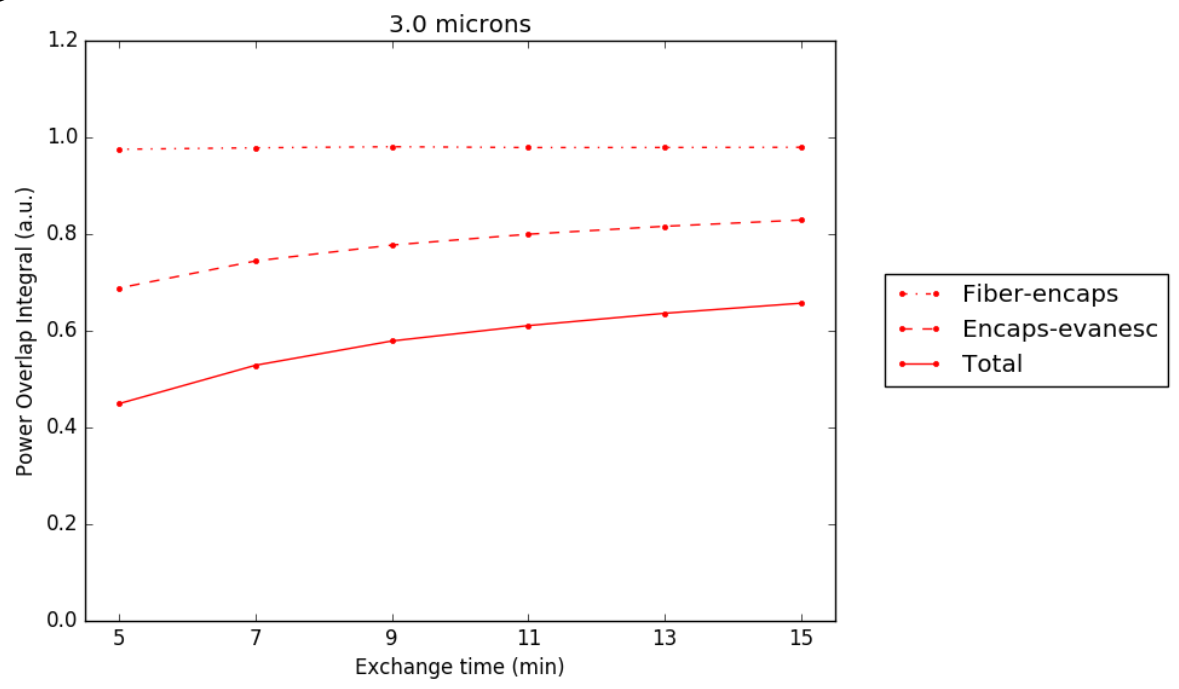

Figure 8. Field overlap integrals of device transitions at $\lambda=800 \mathrm{~nm}$. Waveguide aperture is $3 \mu \mathrm{m}$.

We observe very good coupling between the optical fibers and the encapsulated structure. Field overlapping between the encapsulated and evanescent waveguide is lower and decreases with exchange duration. Figure 9 shows total losses as a function of waveguide fabrication parameters.

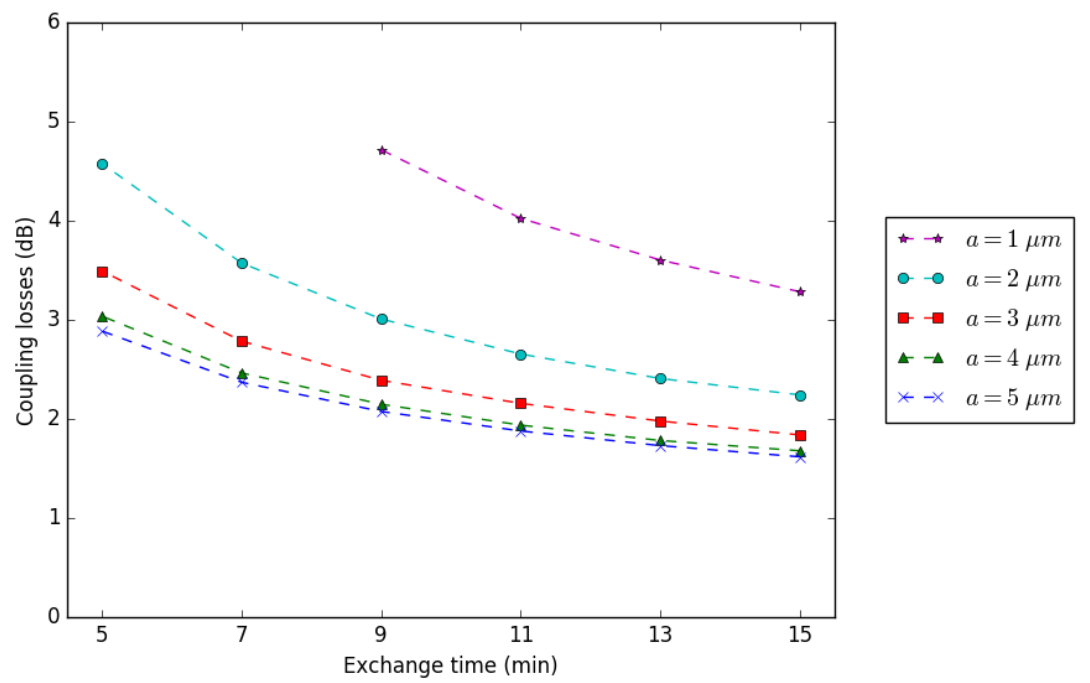

Figure 9. Device total coupling losses at $\lambda=800 \mathrm{~nm}$ as a function of waveguide aperture and exchange time.

Results show lesser coupling losses with higher waveguide apertures and exchange duration. They also show rather similar behavior of losses for apertures of $3 \mu \mathrm{m}$ and higher. Overall intrisinc coupling losses are sufficiently low to expect adequate chip output signal. 


\subsection{Choice of waveguide design}

A waveguide of $\mathrm{a}=3 \mu \mathrm{m}$ in aperture exchanged $7 \mathrm{~min}$ at $353^{\circ} \mathrm{C}$ was chosen to provide single-mode propagation in both the evanescent and encapsulated part of the device. Interaction coefficient is estimated at $0.20 \%$ with coupling losses of $2.8 \mathrm{~dB}$. This should allow for detection of $[\mathrm{Nd}]>6.10^{-2} \mathrm{M}$ and $[\mathrm{Pu}(\mathrm{VI})]>4.10^{-3} \mathrm{M}$ for a device with a $2 \mathrm{~cm}$ interaction length and a system detection limit of $5.10^{-3}$ OD. Figure 10 shows the simulated refractive index profiles of the chosen and their fundamental modes.
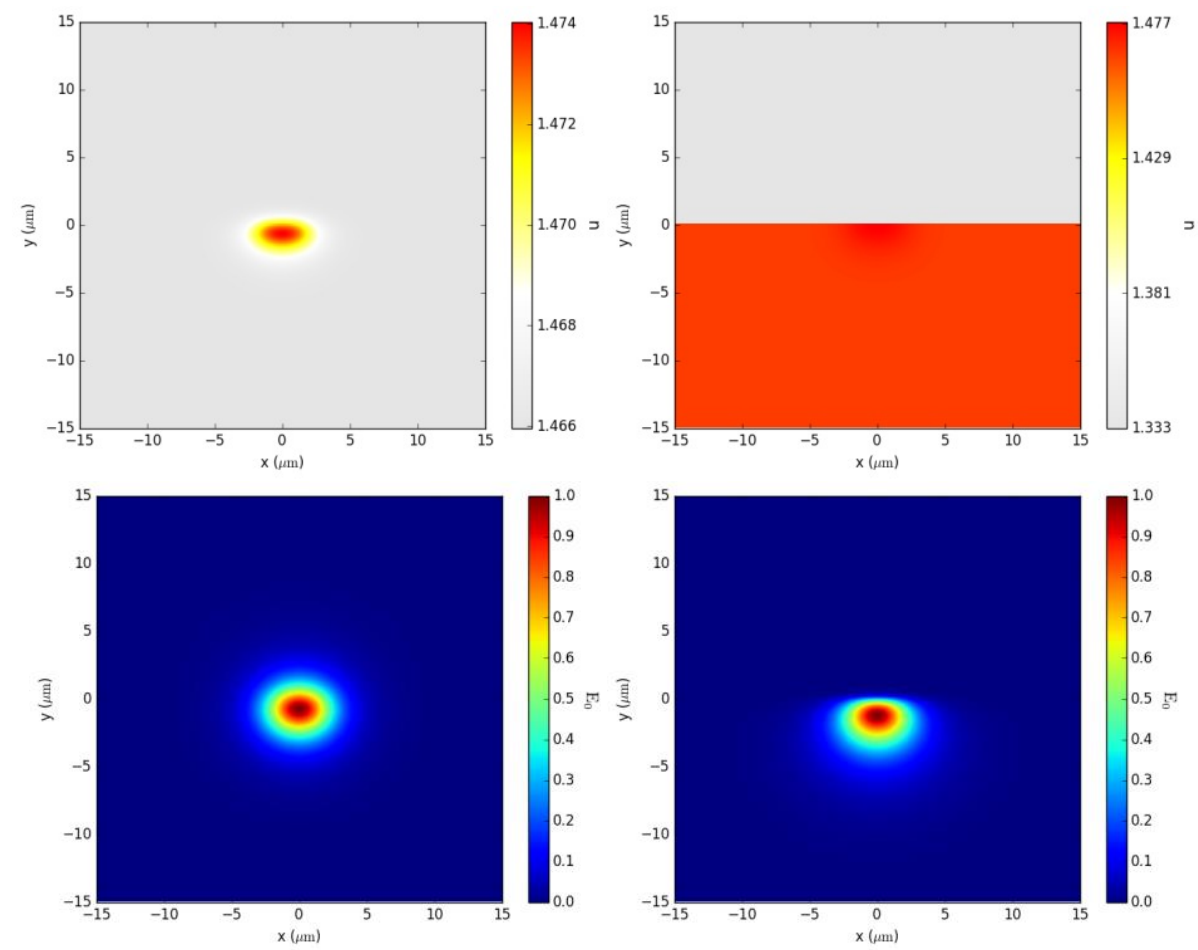

Figure 10. Simulated refractive index profiles (top) and fundamental modes (bottom) of an encapsulated waveguide (left) and evanescent waveguide (right) at $\lambda=800 \mathrm{~nm}$. Ion-exchange parameters are $\mathrm{a}=3 \mathrm{um}, \mathrm{t}=7 \mathrm{~min}, \mathrm{~T}=353^{\circ} \mathrm{C}$ and bonding process annealing parameters are $\mathrm{t}=72 \mathrm{~h}$ and $\mathrm{T}=150^{\circ} \mathrm{C}$.

\section{SENSOR FABRICATION AND CHARACTERIZATION}

The optical wafer is elaborated with the ion-exchange. The optical waveguide is obtained by first depositing an $\mathrm{Al}_{2} \mathrm{O}_{3}$ masking layer on the substrate surface. Waveguide cores are patterned up in the masking layer with photolithograpy. The substrate is then plunged into a molten salt bath in order to proceed to the ion exchange. On the other hand, the fluidic wafer is obtained with a wet HF etching process, described in detail in previous work ${ }^{7}$. The microfluidic channel allows interaction of $2 \mathrm{~cm}$, with a channel depth of $150 \mu \mathrm{m}$. Both wafers are molecularly bonded in order to obtain the final structure ${ }^{8}$.This consists in the chemical activation of the wafer surfaces and structure annealing after contact in order to strengthen the bond. This protocol was adapted from a microelectronic direct wafer-to-wafer bonding technology in order to provide sturdy epoxy-free and glue-free borosilicate glass wafer bonding. Thermal annealing of the structure is performed at low temperatures in order to avoid waveguide core ions diffusion. At this stage, the wafers are diced and optical input and output edges are polished in order to minimize chip insertion losses due to reflections. The device's output mode profile was measured in order to assess simulation results' viability and number of modes. A Thorlabs laserdiode emitting at $780 \mathrm{~nm}$ was used as light source for the device. Mode profile shown in Figure 11 was measured with an IR camera by using an infinity corrected objective focused on the output edge. 


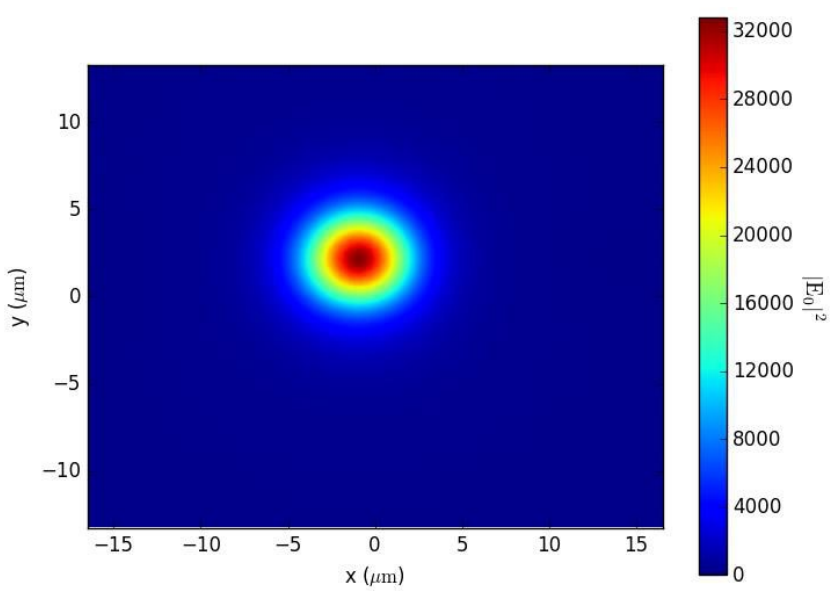

Figure 11. Device output mode profile measurement at $780 \mathrm{~nm}$.

Single-mode behavior is obtained at $780 \mathrm{~nm}$. Guided mode beam-width characteristics $\mathrm{w}\left(1 / \mathrm{e}^{2}\right)$ has been obtained by means of a Gaussian fitting.

Table 1 shows mode-width characteristics of the experimental and simulated output mode.

\begin{tabular}{rcc}
\hline & $\begin{array}{c}\text { Simulated } \\
\text { mode }\end{array}$ & $\begin{array}{c}\text { Measured } \\
\text { mode }\end{array}$ \\
\hline $\mathrm{w}_{\text {hor }}(\mu \mathrm{m})$ & 5.1 & $6.3 \pm 0.4$ \\
$\mathrm{w}_{\text {vert }}(\mu \mathrm{m})$ & 1.9 & $2.8 \pm 0.2$ \\
$\mathrm{w}_{\text {vert- }}(\mu \mathrm{m})$ & 2 & $2.9 \pm 0.2$ \\
\hline
\end{tabular}

Table 1. Simulated and measured mode fitted waist at $\lambda=780 \mathrm{~nm}$ of $3 \mu \mathrm{m}$ aperture encapsulated waveguide ion-exchanged $7 \mathrm{~min}$ at $353^{\circ} \mathrm{C}$ and annealed $72 \mathrm{~h}$ at $150^{\circ} \mathrm{C}$. "hor", "vert+" and "vert-" indices refer to the horizontal, upper vertical and lower vertical waist measurements of the fitted mode distributions.

The experimental results are slightly higher than simulation results, probably due to the fact the bonding annealing ramp in temperature is not taken into account. Successively, device spectral transmission was carried out by injecting a broadband light from a halogen source into the waveguide. Cut-off wavelength was measured by means of an optical spectrum analyzer (Princeton Spectra Pro SP2300). Figure 12 shows a cut-off wavelength between 950 and $1000 \mathrm{~nm}$.

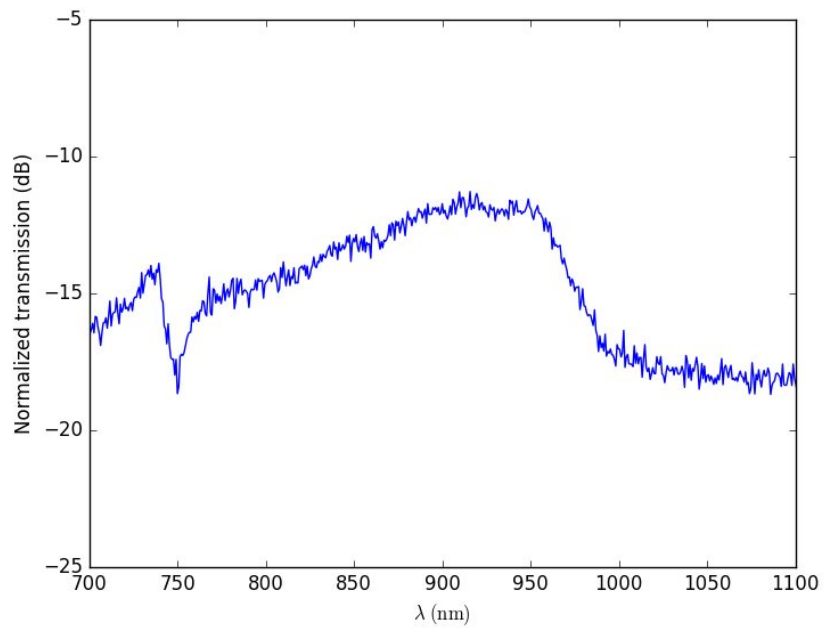

Figure 12. Spectral transmission of the elaborated device. 


\section{PACKAGED DEVICE BENCH TEST}

A stainless steel microfluidic chip mount was fabricated to reach compatibility with commercial interfacing solution (Dolomite microfluidic). The device is connected to capillaries thanks to seals. This allows leak-free pumping of the fluid through external capillaries into the chip and out again. Optical fibers were pigtailed to the integrated waveguide thanks to glass ferules and UV glue according to Figure 13. Such a system allows separating optical pigtailing UV glue from harmful analyte fluids.

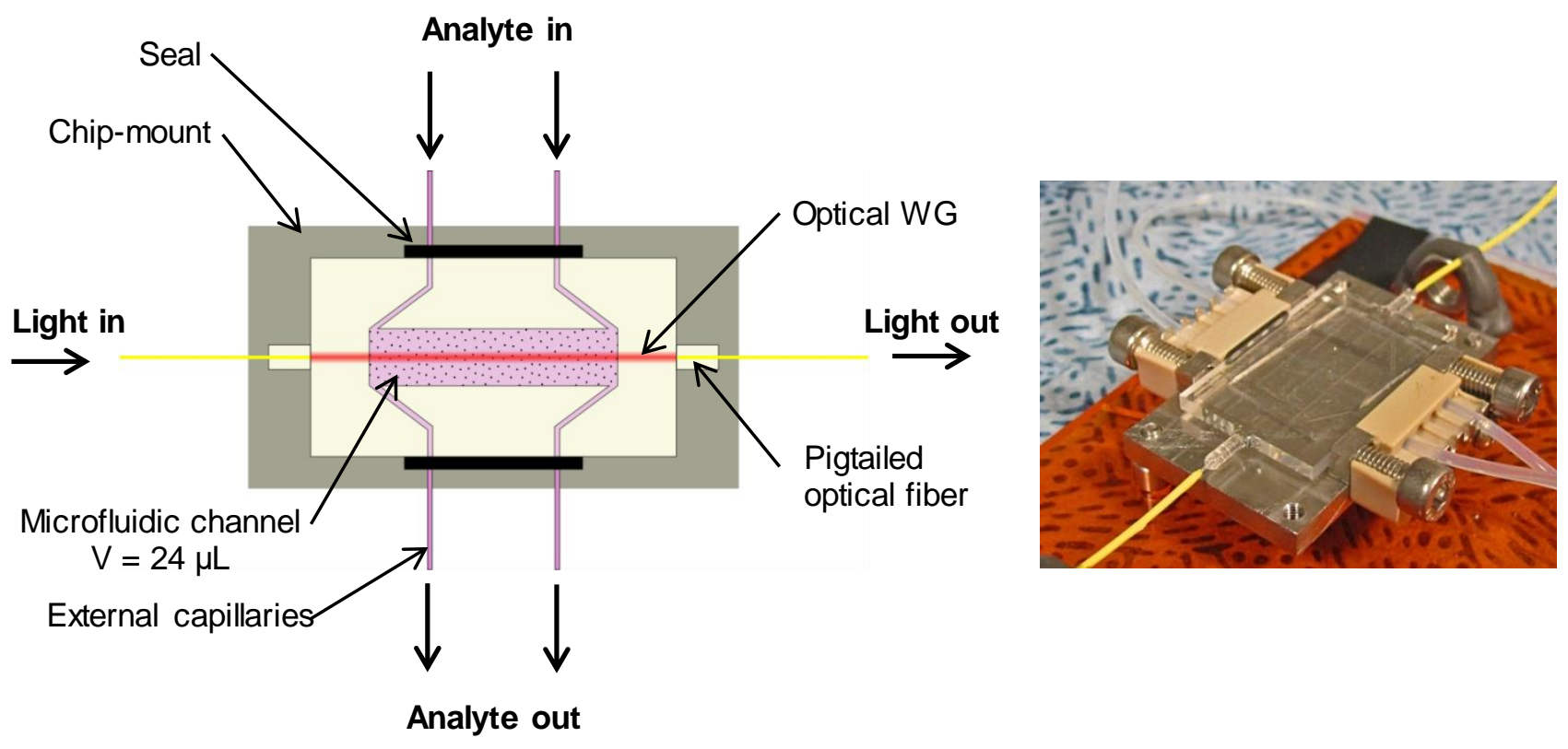

Figure 13. Device fluidic and optical packaging for deported use.

Total injection losses were measured at $12 \pm 2 \mathrm{~dB}$ on a range of 5 standardized devices fabricated within several technological runs. Experimental losses are higher than the intrinsic coupling losses simulated above due to optical connector losses, propagation losses and technological imperfections.

Chemical characterization was carried out by measuring absorption peak as a function of Nd concentration. A fluid multiplexer and solenoid valves (Elveflow MUX) were used to automate fluid handling and to allow sequential fluid injection. A SLED (Exalos EXS210025-01) light source centered at 790nm with a 20nm bandwidth is connected at the device input using single mode fiber. The $\mathrm{Nd}$ absorption band at $794 \mathrm{~nm}$ is monitored at the output by recording the spectrum on a cooled CCD camera (Princeton Instruments PIXIS 400) at the output of a spectrometer (Princeton instruments SP2300) connected to the chip with a multimode fiber. The neodymium absorption is analyzed by alternating rinsing and filling cycles as shown in Figure 14.

Several solutions of Nd concentrations ranging from 0.12 mol. $\mathrm{L}^{-1}$ to $1 \mathrm{~mol} . \mathrm{L}^{-1}$ in $1 \mathrm{~mol} . \mathrm{L}-1$ nitric acid have been studied. A 1 mol.L $\mathrm{L}^{-1}$ nitric solution is used for rinsing and a reference spectrum is recorded. Then the microfluidic channel is filled with analyte solution and an absorption spectrum is recorded. The absorbance is calculated accordingly to equation (1). For each concentration the rinsing/filling cycles have been repeated three times. The absorbance is averaged over these three measurements. The obtained spectra are reported in Figure 15 and show an increase in measured $\mathrm{Nd}$ absorbance with $\mathrm{Nd}$ concentration. 


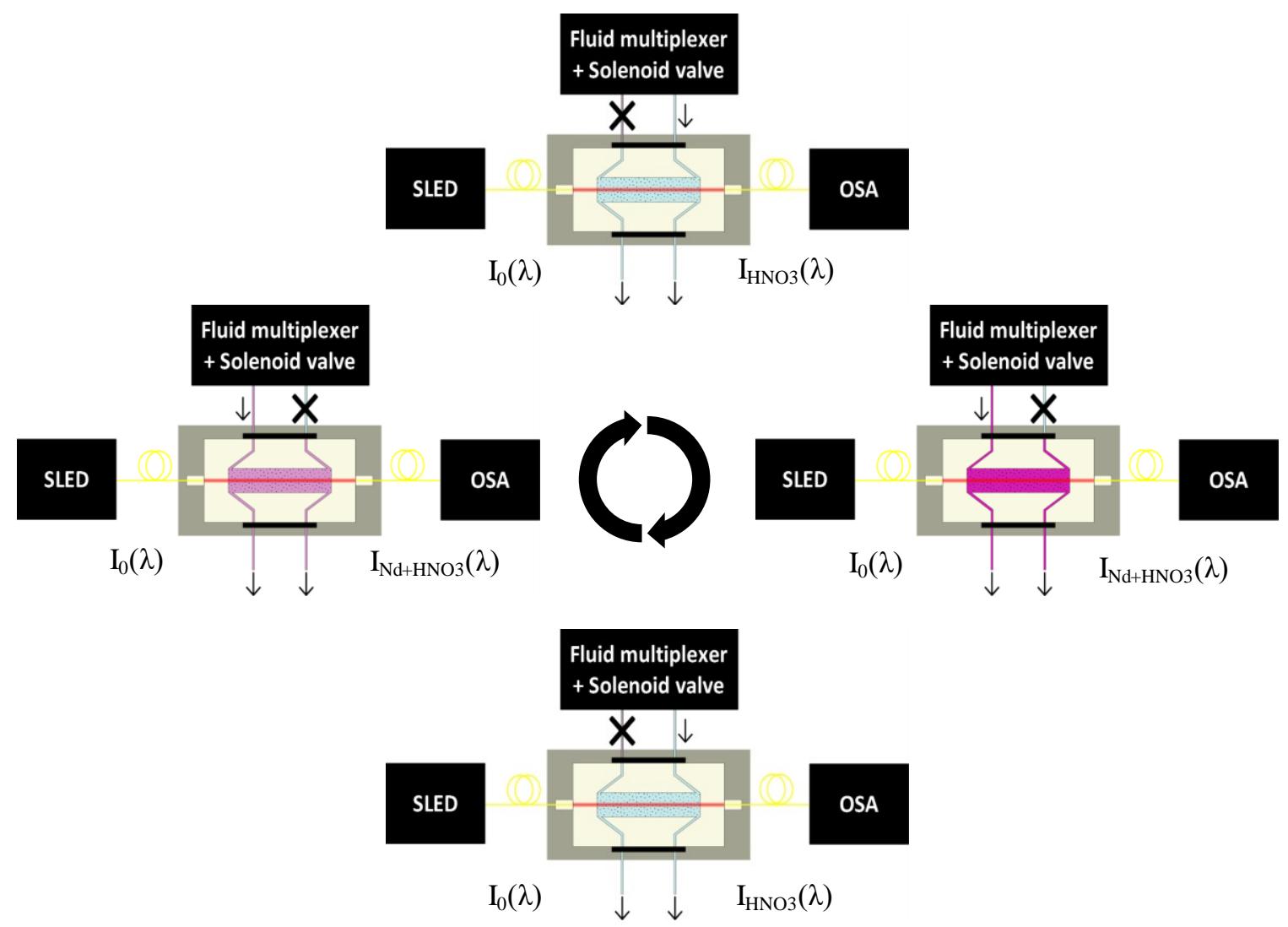

Figure 14. Bench test measurement principle. Cycles of reference nitric acid and concentrated Nd in nitric acid fluid injection were automated to measure spectral response of the difference solutions.

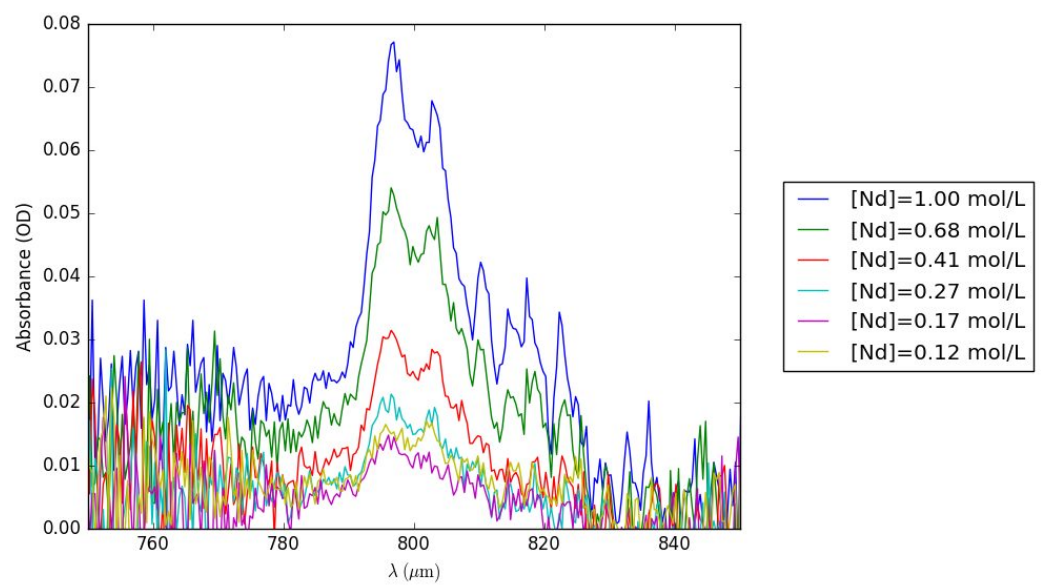

Figure 15. Absorbance measurements of $\mathrm{Nd}$ in $1 \mathrm{M} \mathrm{HNO}_{3}$ at different concentrations

The absorbance value at $794 \mathrm{~nm}$ is extracted from previous spectrum and plotted in Figure 16 according to the neodymium concentration. We notice the good response linearity over the concentrations range under study. This corresponds to an experimental interaction coefficient value of $0.4 \%$ using equation (1) according to Figure. This result is slightly higher than the simulated value probably due to the fact that $\mathrm{Nd}$ solutions have slightly higher refractive index than the simulated reference solution. This measurement was repeated at 3 days of interval and shows reproducibility in time. 


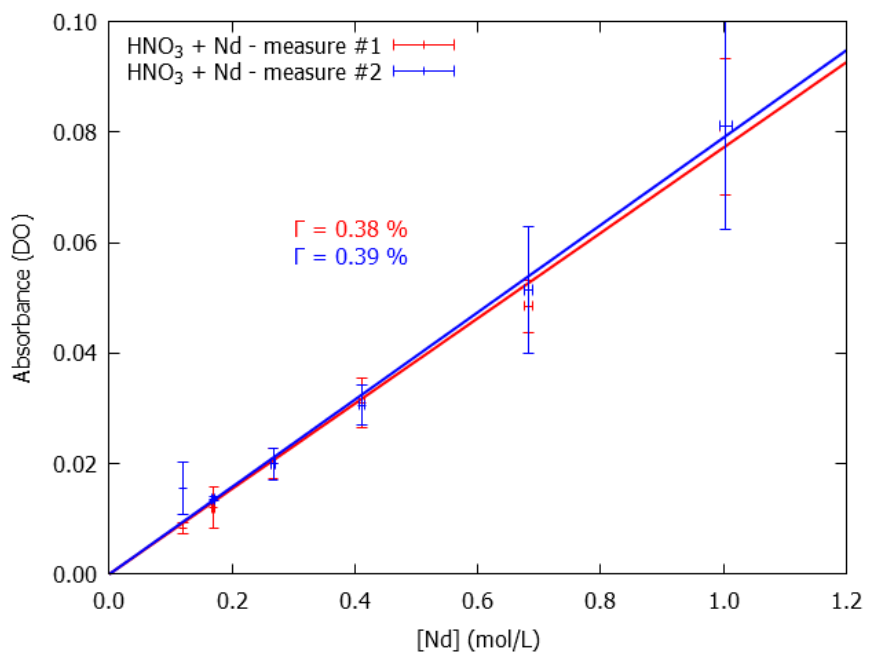

Figure 16. Device absorbance vs. [Nd] calibration. Two measurements carried out at a 3 day interval are displayed.

\section{FIRST NUCLEARIZED RESULTS AND PERSPECTIVES}

The sensor behavior has been verified in non-radioactive conditions. In order to test the device in nuclear conditions, the characterized chip was transferred to a nuclear glove-box depicted in Figure 17. The device was connected to the light source and spectrometer through optical connector bulkheads in the glove-box walls. Because of the harsh environment, a simpler fluid handling solution has been used. Analyte solution containing $1.42 \mathrm{~mol} . \mathrm{L}^{-1}$ neodymium and rinsing 1 mol. $\mathrm{L}^{-1}$ nitric acid were injected thanks to capillaries and syringes. In this case, a single concentration has been tested. The $\mathrm{Nd}$ absorbance spectrum is then normalized with the concentration and interaction coefficient to obtain the molar extinction coefficient, as reported in Figure 17.

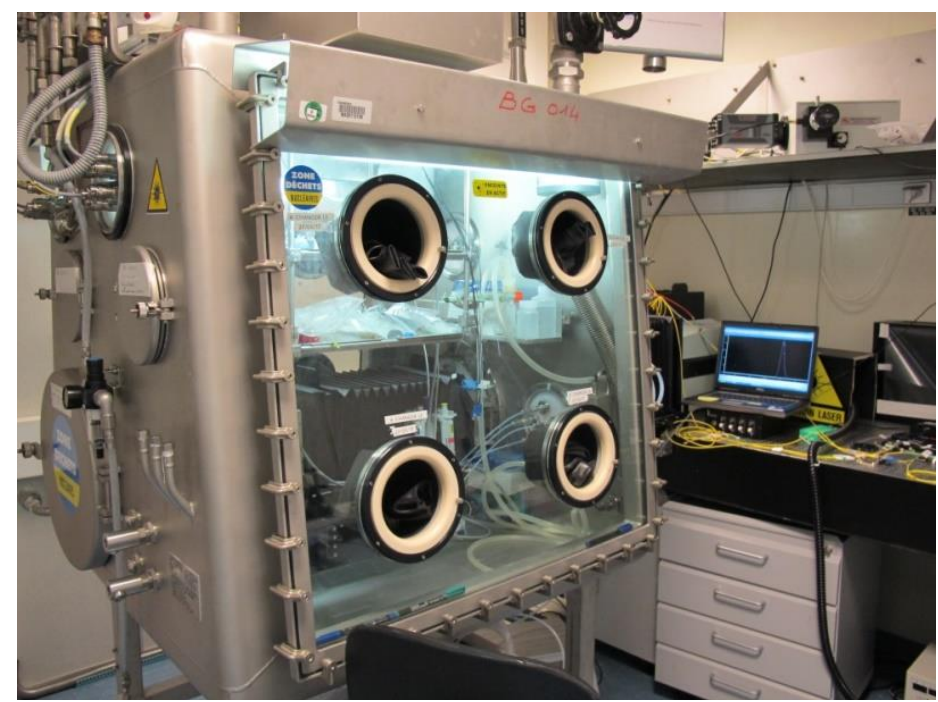

Figure 17. Nuclearized measurement set-up with the probing device inside a glove-box.

The extinction coefficient has been previously determined using the same solution with a commercially available spectrometer (Shimadzu UV-1800) and superimposed in Figure 18. The comparison to the measured spectrum in the glove-box highlights a good agreement. The latest proves the feasibility of measurement in nuclear conditions using a microsystem. Indeed, the glove-box exhibits both radioactive and corrosive atmosphere because of past activities. 


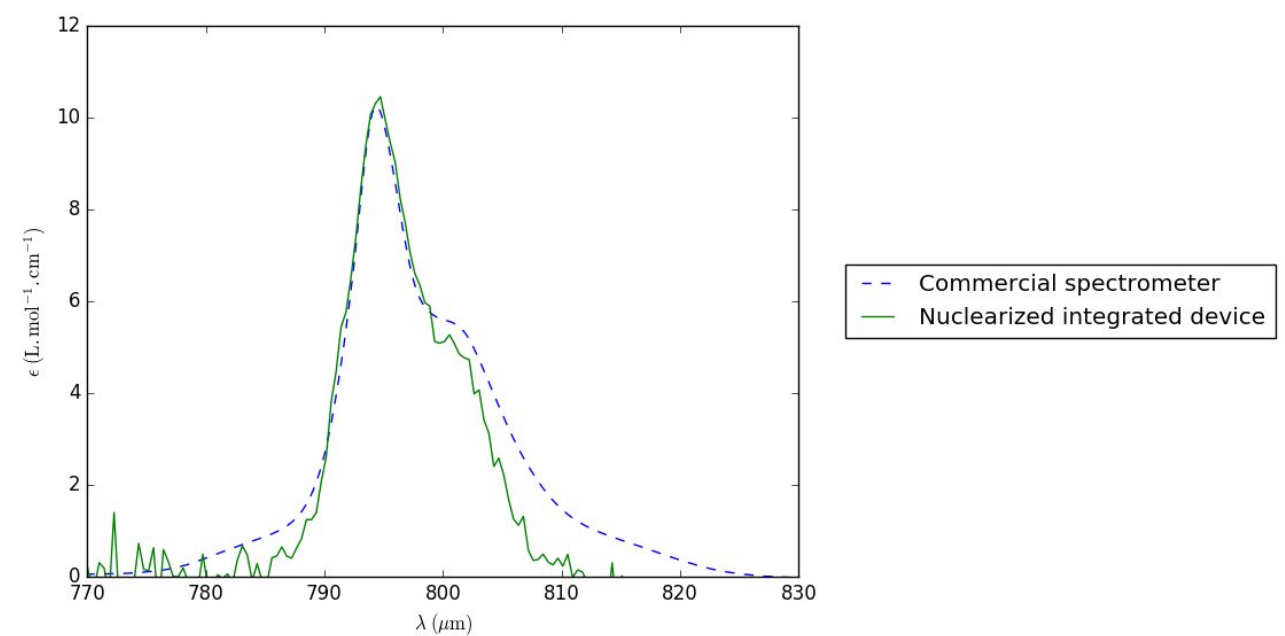

Figure 18. Nd absorption peak at $794 \mathrm{~nm}$ measured with the nuclearized integrated device and a commercial Shimadzu spectrometer.

Having demonstrated that the elaborated device allows chemical concentration measurements, advanced sensor calibration and metrology is underway in order to allow industrial use. In particular the non-linearity of the interaction coefficient that is a function of solution refractive index and wavelength will be studied. Future development steps of this work include the implementation of state-of-the-art sensing designs to enhance sensor sensitivity for which two solutions will be investigated: a nano-fluidic hybrid waveguide allowing to increase interaction coefficient and the integration of spiral waveguides to increase interaction length. Finally, measurement of plutonium solutions is underway.

\section{CONCLUSION}

We have shown successful integration of both fluidic and optical functions on a glass chip. Sensor engineering was carried out in order to provide microfluidic chemical analyses suited for nuclear manipulation. The means of probing consists in absorption spectroscopy that is versatile, label-free and particularly adapted to the sought-out application. Optical behavior of the device was tailored by computer simulations and verified experimentally. Device packaging was elaborated in order to allow for remote measurements. Approach validation was obtained by obtaining reproducible neodymium absorption measurements and repeating the analysis inside a nuclear glove-box with the same device. Measurements of plutonium solutions are underway and sensor sensitivity enhancement is being studied.

\section{ACKNOWLEDGMENTS}

The authors thank G. Grosa and L. Guillerme for their technical support and would like to acknowledge L. Bastard for the use of his software.

\section{REFERENCES}

[1] Janssens-Maenhout, G., Buyst, J.., Peerani, P., "Reducing the radioactive doses of liquid samples taken from reprocessing plant vessels by volume reduction," Nucl. Eng. Des. 237(8), 880-886 (2007).

[2] Moulin, C., Briand, A., Decambox, P., Fleurot, B., Lacour, J. L., Mauchien, P.., Remy, B., 'Techniques d'analyses d'actinides et de radioéléments d'intérêt par spectroscopie laser," Radioprotection 29, 517-538 (1994).

[3] Hill, K. O., Watanabe, A.., Chambers, J. G., "Evanescent-Wave Interactions in an Optical Wave-Guiding Structure," Appl. Opt. 11(9), 1952-1959 (1972). 
[4] Pandraud, G., Koster, T. M., Gui, C., Dijkstra, M., van den Berg, A.., Lambeck, P. V., "Evanescent wave sensing: new features for detection in small volumes," Sens. Actuators Phys. 85(1-3), 158-162 (2000).

[5] Broquin, J.-E., "Glass integrated optics: state of the art and position toward other technologies," Proc. SPIE 6475, 647507-647513 (2007).

[6] Tervonen, A., West, B. R.., Honkanen, S., "Ton-exchanged glass waveguide technology: a review," Opt. Eng. 50(7), 71107-71115 (2011).

[7] Allenet, T., Bucci, D., Geoffray, F., Canto, F., Couston, L., Jardinier, E.., Broquin, J.-E., "Packaged integrated opto-fluidic solution for harmful fluid analysis," Proc. SPIE 9750, 975000-975011 (2016).

[8] Xu, Y., Wang, C., Li, L., Matsumoto, N., Jang, K., Dong, Y., Mawatari, K., Suga, T.,, Kitamori, T., "Bonding of glass nanofluidic chips at room temperature by a one-step surface activation using an $\mathrm{O} 2$ /CF 4 plasma treatment," Lab. Chip 13(6), 1048-1052 (2013). 